\title{
A feasibility study of photoacoustic imaging of ex vivo endoscopic mucosal resection tissues from Barrett's esophagus patients
}

(9)(९

\author{
Authors \\ DaCosta ${ }^{1}$, Brian C. Wilson ${ }^{1}$ \\ Institutions \\ 1 Princess Margaret Cancer Centre, Toronto, Ontario, \\ Canada \\ 2 St. Michael's Hospital, Toronto, Ontario, Canada
}

Liang Lim², Catherine J. Streutker², Norman Marcon², Maria Cirocco², Alexandra Lao², Vladimir V. lakovlev², Ralph

submitted 20.12.2016

accepted after revision 2.5.2017

Bibliography

DOI https://doi.org/10.1055/s-0043-111790 |

Endoscopy International Open 2017; 05: E775-E783

(c) Georg Thieme Verlag KG Stuttgart · New York ISSN 2364-3722

Corresponding author

Liang Lim, PhD, University Health Network - Princess

Margaret Cancer Centre, 101 College Street,

PMCRT \#15-301V Toronto, Ontario M5G 1L7, Canada

samlim@utexas.edu

\section{ABSTRACT}

Background and study aims Accurate endoscopic detection of dysplasia in patients with Barrett's esophagus (BE) remains a major clinical challenge. The current standard is to take multiple biopsies under endoscopic image guidance, but this leaves the majority of the tissue unsampled, leading to significant risk of missing dysplasia. Furthermore, determining whether there is submucosal invasion is essential for proper staging. Hence, there is a clinical need for a rapid in vivo wide-field imaging method to identify dysplasia in BE, with the capability of imaging beyond the mucosal layer. We conducted an ex vivo feasibility study using photoacoustic imaging (PAI) in patients undergoing endoscopic mucosal resection (EMR) for known dysplasia. The objective was to characterize the esophageal microvascular pattern, with the long-term goal of performing in vivo endoscopic PAl for dysplasia detection and therapeutic guidance.

Materials and methods EMR tissues were mounted luminal side up. The tissues were scanned over a field of view of $14 \mathrm{~mm}$ (width) by $15 \mathrm{~mm}$ (depth) at 680,750, and $850 \mathrm{~nm}$ ( $40 \mathrm{MHz}$ acoustic central frequency). Ultrasound and photoacoustic images were simultaneously acquired. Tissues were then sliced and fixed in formalin for histopathology with hematoxylin and eosin staining. A total of 13 EMR specimens from eight patients were included in the analysis, which consisted of co-registration of the photoacoustic images with corresponding pathologist-classified histological images. We conducted mean difference test of the total hemoglobin distribution between tissue classes.

Results Dysplastic and nondysplastic BE can be distinguished from squamous tissue in $84 \%$ of region-of-interest comparisons (42/50). However, the ability of intrinsic PAI to distinguish dysplasia from NDBE, which is the clinically important challenge, was only about $33 \%$ (10/30).

Conclusion We demonstrated the technical feasibility of this approach. Based on our ex vivo data, changes in total hemoglobin content from intrinsic PAI (i.e. without exogenous contrast) can differentiate $B E$ from squamous esophageal mucosa. However, most likely intrinsic PAI is unable to differentiate dysplastic from nondysplastic BE with adequate sensitivity for clinical translation.

\section{Introduction}

In North America approximately 19000 new cases of esophageal cancer are diagnosed annually. The 5-year survival rate is only about $15 \%$, with 18000 cancer-related deaths annually $[1,2]$. The most important risk factor for esophageal adenocarcinoma is Barrett's esophagus (BE), which affects some 3.5 mil- lion adults in North America. This is a condition in which the outermost layer of esophageal squamous mucosa (whitish in appearance on standard endoscopy) transforms to gastric/ intestinal-type mucosa (pink-red appearance) due to chronic gastroesophageal reflux. Patients with BE have a $>30$-fold increased risk of developing esophageal adenocarcinoma [3]. 
The current clinical standard for esophageal cancer diagnosis in patients with BE is targeted biopsies of focal visible abnormalities followed by 4-quadrant random biopsy every 1 $2 \mathrm{~cm}$ along the length of the Barrett's segment under whitelight endoscopic guidance. However, the majority of the tissue remains unsampled, leading to significant risk of missing dysplasia, especially in areas with no visible abnormalities. Biopsied $B E$ tissue is then staged by histopathology: in order of cancer progression, from nondysplastic BE (NDBE), to low grade dysplasia (LGD), high grade dysplasia (HGD) and, ultimately, cancer (e.g. adenocarcinoma). Whereas less than $10 \%$ of $\mathrm{BE}$ patients progress to HGD or cancer, patients with HGD are at considerably increased risk of progressing to cancer [4], with yearly rates of $4 \%-11.8 \%[5,6]$.

Early detection of dysplasia, especially HGD, is crucial in managing these patients. Newer methodologies such as cytology from brushes [7] and sponges [8] are being developed but are not yet in common usage. Several clinical trials with a variety of chromoendoscopy have shown promising results [9]. However, chromoendoscopy only enhances the surface features of the tissue and does not provide submucosal information. As well as detecting HGD and intramucosal adenocarcinoma, determining whether there is submucosal invasion is essential for proper staging. Thus, the absence of submucosal invasion indicates that minimally invasive endoscopic mucosal resection (EMR) is potentially curative, whereas its presence indicates the need for esophagectomy, which results in significant mortality as well as detriment to quality of life. Hence, there is an unmet need for a rapid, wide-field endoscopic imaging method to identify dysplasia in BE, with the capability of imaging beyond the mucosal layer.

A wide variety of optical imaging and spectroscopic techniques have been investigated to address this need, including diffuse reflectance spectroscopy [10], tissue autofluorescence [11], contrast-based fluorescence [12], Raman spectroscopy [13], optical coherence tomography [14,15], and narrow-band imaging [16], as well as various combinations of these techniques [17]. However, the clinical need is still largely unmet: for example, optical coherence tomography looks promising in providing large-area scanning at high spatial resolution, but is marginal in terms of depth $(\sim 1.5-2 \mathrm{~mm})$ to reliably assess submucosal invasion.

The mechanistic rationale for using photoacoustic imaging (PAI) in BE is that studies using narrow-band imaging [16] or diffuse reflectance spectroscopy [10] have reported microvasculature changes between NDBE and dysplasia, and this is consistent with angiogenesis in tumor progression [18,19]. PAI, based on the detection of acoustic waves generated by local absorption of short ( ns) pulses of laser light, is specifically sensitive to detecting blood vessels in tissue as a result of light absorption by haemoglobin, and is also able to image much deeper into tissue (up to $3.5 \mathrm{~cm}$ ) [20] than other optical endoscopic imaging techniques [21]. Hence, PAI has the potential to provide imaging of microvasculature from the tissue surface to well below the mucosal layer.

We hypothesize that PAI can detect microvasculature changes between nondysplastic and dysplastic BE. Here, we report a feasibility study using PAI in ex vivo tissues from BE patients with known dysplasia who were undergoing EMR. We have previously reported the technical feasibility of this technique [22], and here we provided analysis from our first group of patients. This analysis consisted of acoustic image co-registration with pathologist-annotated histological images, followed by mean difference comparison between the intrinsic PAl intensity images of the EMR specimen and the pathological classification. Initial results show clearly that intrinsic PAI can differentiate BE from squamous tissue $(42 / 50=84 \%)$, but is likely not sufficiently sensitive to diagnose dysplasia in BE.

\section{Materials and methods}

\section{Photoacoustic system}

We used a commercial photoacoustic tomography imaging system (Vevo LAZR Visualsonics, Toronto, Ontario, Canada), which has been described in detail elsewhere [23]. This included an integrated fiberoptic linear-array transducer (LZ550, Visualsonics, $40 \mathrm{MHz}$ central acoustic frequency, 256 elements) and a crossed-laser-beam geometry for optical excitation [23]. Photoacoustic and ultrasound images were collected at a frame rate of $5 \mathrm{~Hz}$. The linear transducer generated images with a field of view of approximately $15 \mathrm{~mm}$ (depth) by $14 \mathrm{~mm}$ (width). The PAl system was located adjacent to a clinical endoscopy suite, so that tissue specimens could be imaged immediately after endoscopic resection.

\section{Patient enrollment}

All patients were scheduled for routine screening, diagnostic, and/or therapeutic endoscopy at the Gastroenterology Clinic, St. Michael's Hospital, Toronto, Canada. Patients were 18 years or above, able to sign the informed consent, and had known pathology indicative for EMR. We excluded patients with any underlying medical condition contraindicating EMR or who had gastrointestinal (Gl) malignancy treated with chemotherapy or radiotherapy within the past 3 years. All patients provided written informed consent prior to their endoscopy procedure. The study was carried out with institutional Research Ethics Board approval.

Eight patients were enrolled in this initial feasibility study, from whom PAI and ultrasound scans were obtained in 13 ex vivo EMR tissue samples. Patients' pre-EMR diagnosis ranged from inflamed mucosa to intramucosal carcinoma. Patients' post-EMR diagnosis ranged from NDBE to intramucosal carcinoma. EMRs were directed at areas with field defect most suspicious for disease. Detailed information of pre-EMR diagnosis, endoscopic descriptions, and resected specimen diagnosis are included in the supplementary materials (see S1. Patient List).

\section{EMR tissue preparation}

The study neither changed standard endoscopic procedure nor increased risk to the patients, as the ultrasound and PAI scans were performed on excised tissues. We also confirmed that the imaging procedure had no detectable effect on the tissues and did not compromise subsequent clinical histopathology. When BE with suspected dysplasia is identified during endos- 
copy, patients may undergo an EMR procedure to excise BE tissues for diagnosis. There are variations of EMR procedures [24]. In the current study, all EMRs were performed using a Duette suction device (Duette; Cook Medical, Bloomington, Indiana, USA), which excises the tissue by suctioning to draw the tissue into the cap, followed by ligation (rubber banding) to a polyplike shape and resection using an electrosurgical snare.

\section{Data acquisition}

Excised tissues were mounted using ultrasound gel onto a plastic dish (\#430167; Corning Inc., New York, USA) filled with clear gelatin ( $5 \%$ weight of $40 \mathrm{~mL}$ volume) to minimize acoustic signals from the dish. Digital photographs were taken and included a scale bar. Phosphate buffered saline was then added to the dish to provide good acoustic contact with the transducer and to prevent tissue dehydration. A transducer cover (CIV-Flex 610-637; Civco, lowa, USA) was mounted onto the transducer probe to protect the tissue sample and avoid cross contamination. The transducer was lowered in contact with the phosphate buffered saline and the sample was centered on the transducer field of view. Because of the crossed-laser-beam geometry of the PAI fiber bundle attached to the transducer, there is an optimal photoacoustic zone located between 9 and $11 \mathrm{~mm}$ in front of the probe face. Hence, we positioned the top surface of the tissue at $9 \mathrm{~mm}$ for consistency between samples.

PAl scans were made at each of 680,750 , and $850 \mathrm{~nm}$. Additional measurements were made at 824 and $970 \mathrm{~nm}$ in anticipation of future work in which these data will serve as controls in studies of topical biomarker-targeted porphyrin-lipid nanoparticle contrast $[25,26]$. The transducer was mounted onto a motorized translational stage and scanned over a 3D volume at $\sim 0.06 \mathrm{~mm}$ step size. The time for complete data acquisition over a $17 \mathrm{~mm}$ scan distance was approximately 5 minutes ( $\sim 1$ minute per wavelength). The PAI laser power was below the ANSI maximum permissible exposure for skin $\left(20-70 \mathrm{~mJ} / \mathrm{cm}^{2}\right.$ for $\left.680-970 \mathrm{~nm}\right)$ [27], avoiding any tissue heating that might damage the tissue, as confirmed on subsequent histopathology.

\section{Histopathology procedure}

Once data acquisition was completed, a pathologist marked the orientation of the specimen for subsequent histopathology and sectioning along the ultrasound/photoacoustic imaging plane. Larger samples were grossly cut into $2 \mathrm{~mm}$ thick slabs prior to fine sectioning. Sectioned tissues were assigned a unique identifier and fixed in $10 \%$ formalin. The tissues were kept moist with phosphate buffered saline from the time of excision until fixation in formalin. The whole procedure, from excision to placing the tissue in formalin, was completed within 60 minutes to avoid compromising subsequent clinical histopathology.

\section{Data analysis}

PAI and ultrasound data were analyzed in Matlab (Mathworks; Natick, Massachusetts, USA). The PAI intensities at $750 \mathrm{~nm}$ and $850 \mathrm{~nm}$ were used as input to estimate the relative concentrations of oxy-hemoglobin $\left(\mathrm{HbO}_{2}\right)$ and deoxy-hemoglobin $(\mathrm{Hb})$ using the corresponding known molar extinction coefficients
$\left(\varepsilon_{\mathrm{HbO} 2}\right.$ and $\left.\varepsilon_{\mathrm{Hb}}\right)[23,28,29]$. The relative total hemoglobin $\left(\mathrm{Hb}_{\mathrm{T}}\right)$ was then given by the sum of the relative $\mathrm{HbO}_{2}$ and $\mathrm{Hb}$ values.

A digitized whole-section hematoxylin and eosin-stained slide from each tissue was segmented and color coded by an expert GI pathologist (C.S. and A.L.) according to the following tissue classes: squamous mucosa (blue), inflammation (gray), intestinal metaplasia (IM; green), dysplasia (yellow), and adenocarcinoma (orange). - Fig. 1 shows two typical histological images ( $\triangleright$ Fig.1a,c), overlaid with the color-coded segmentation. The histopathology slices were then co-registered with the closest corresponding ultrasound and photoacoustic image ( $\vee$ Fig. 1b,d), and regions of interest (ROIs) for each tissue class were mapped manually onto these acoustic images. PAI with the above probe-tissue geometry was able to detect signal from blood located up to $2 \mathrm{~mm}$ beneath the tissue surface.

The ROI comparisons were limited to regions within 9$11 \mathrm{~mm}$ from the transducer surface in order to minimize the effect of nonuniform distribution of the excitation light, and regions within the same field of view to minimize the effects of intra- and inter-patient and site heterogeneity in $\mathrm{BE}$, which is known to be high. To make PAI comparison across patients and specimens without accounting for this heterogeneity would be misleading; therefore, histological mapping was done within each histological slice ( $\mathbf{F i g . 1 a , c )}$ and comparisons were made only within the same field of view. Comparisons were made for all possible ROI permutations within each image: for example, for the case shown in $>$ Fig. 1a,b, these were ROI-IM vs. ROI-SQ1, ROI-IM vs. ROI-SQ2, and ROI-SQ1 vs. ROI-SQ2, but the third comparison was not included in the final analysis as both ROI-SQ1 and ROI-SQ2 belonged to the same diagnostic class (squamous).

- Fig. 2 shows an example of comparison between two ROls from > Fig. 1b. Here, ROI-IM (green) had a higher mean relative $\mathrm{Hb}_{\top}$ value than ROI-SQ1 (blue), the difference being statistically significant (Wilcoxon Rank Sum test, $P<0.05$ ). We considered ROI-IM to have higher relative $\mathrm{Hb}_{T}$ than that of ROI-SQ1 when the mean $\mathrm{Hb}_{\mathrm{T}}$ was higher and the difference in their $\mathrm{Hb}_{\mathrm{T}}$ distributions was statistically significant $(P<0.05)$. If these conditions did not hold, then the relative $\mathrm{Hb}_{\mathrm{T}}$ comparison for that ROI pair was considered to be negative.

\section{Results}

- Fig. 3 shows $3 \mathrm{D}$ reconstruction of a complete EMR tissue volume using the acoustic images from a typical EMR tissue. Ultrasound ( Fig.3a)provided structural information, while PAI ( $\triangleright$ Fig. $\mathbf{3 b}, \mathbf{c}$ ) provided complementary information about the distribution of absorbers (i.e. blood) within the tissue.

The ROI comparisons of relative $\mathrm{Hb}_{\mathrm{T}}$ are summarized in - Table 1. In general, the relative $\mathrm{Hb}_{\mathrm{T}}$ differences between the subclasses of BE (dysplasia, NDBE with inflammation, and IM) were not consistent. However, relative $\mathrm{Hb}_{T}$ was higher in $\mathrm{BE}$ than in squamous mucosa in the majority of comparisons.

Based on the relative $\mathrm{Hb}_{\mathrm{T}}$ content, NDBE and BE can be distinguished from squamous tissue in $84 \%$ of ROI comparisons (42/50). This is not surprising, as the chronic inflammation and resulting mucosal transformation in $\mathrm{BE}$ is associated with 

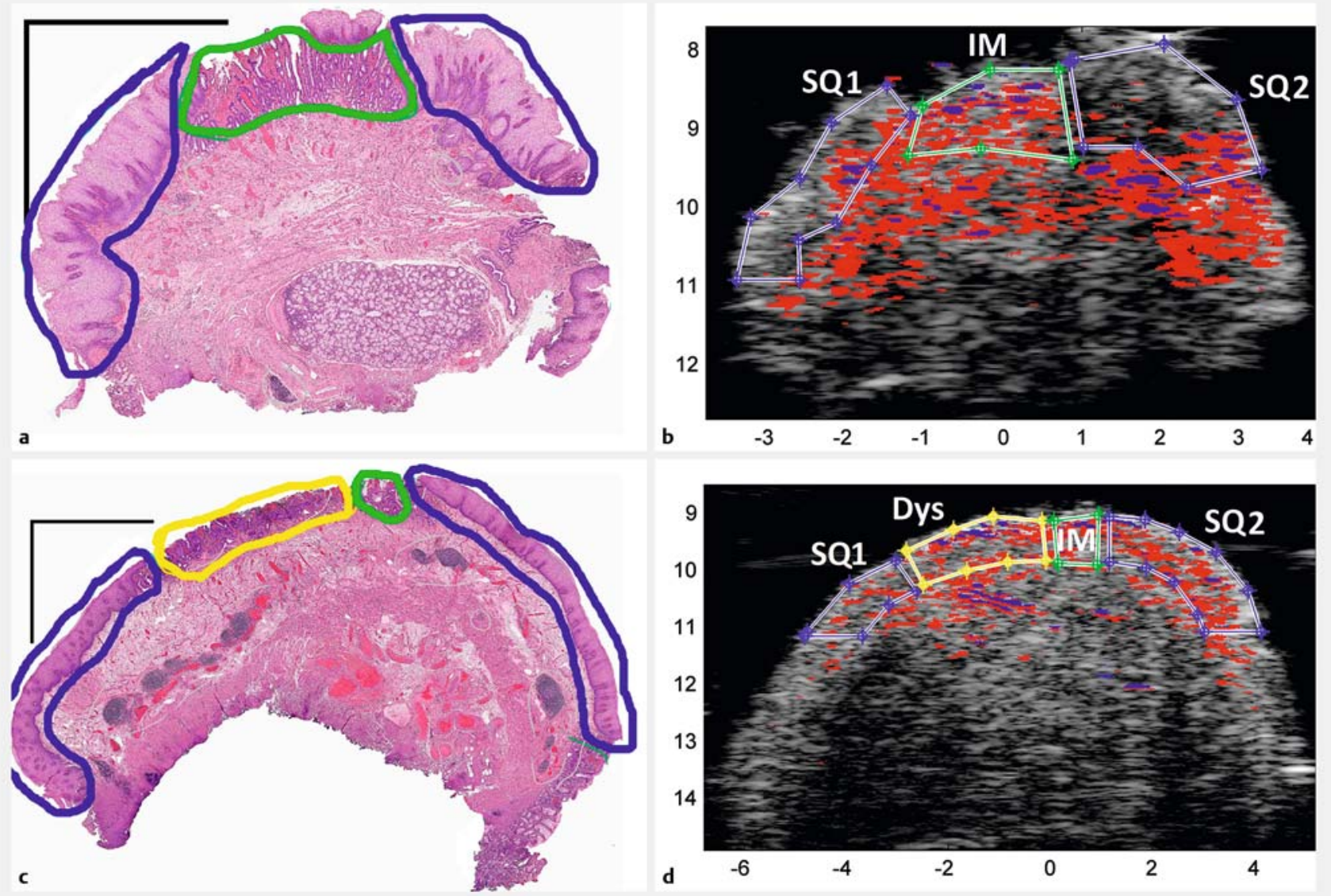

- Fig. 1 Two examples of co-registered histopathology (hematoxylin and eosin staining) and ultrasound/photoacoustic images. a, c Histopathology overlaid with color-coded tissue classification (green: intestinal metaplasia [IM]; blue: squamous [SQ]; yellow: dysplasia [Dys]; gray: inflammation). The black lines are $2 \mathrm{~mm}$ scale bars. b, $\mathbf{d}$ Corresponding ultrasound (grayscale) and photoacoustic images (blue: $750 \mathrm{~nm}$; red $850 \mathrm{~nm}$ ). Regions of interest for different tissue classes are indicated. Scales are in $\mathrm{mm}$.

increased vascularity, as evidenced by the marked color difference between $B E$ and squamous mucosa visible on white-light endoscopy. However, the ability of intrinsic PAl to distinguish dysplasia from NDBE, which is the clinically important challenge, was only about $33 \%$ (10/30), even though the wavelengths used were hemoglobin sensitive so that PAI was expected to pick up alterations in the tissue microvascular density.

\section{Discussion and conclusions}

In terms of technical issues in performing these ex vivo PAI scans, as previously mentioned the crossed-laser-beam geometry of the PAI probe resulted in an optimal photoacoustic zone within the field of view located between 9 and $11 \mathrm{~mm}$ from the probe surface. This raised challenges in both data acquisition and analysis. For data acquisition, accurate placement of the tissue sample within the field of view is required to acquire consistent PAI signals from the same tissue volume. However, this is difficult to achieve because the EMR tissue ligation results in a domed or polyp-like specimen shape. Furthermore, the sizes of the EMR tissue samples were highly variable, from $<3 \times 3 \times$ $2 \mathrm{~mm}^{3}$ up to $10 \times 12 \times 6 \mathrm{~mm}^{3}$. Given these constraints, the sim- plest and most reliable solution was to position the top of the tissue at $9 \mathrm{~mm}$. For data analysis, the crossed-beam geometry has higher spatial variation in intensity than a flat-top beam. Even with simpler beam geometry, the relationship of photoacoustic signal to physiologically relevant parameters is complex, and estimating the hemoglobin-related concentrations is not strictly quantitative, as documented in other studies [3032]. Hence, as an initial analysis without requiring complex inverse modeling, we included only ROls lying within the optimal (9-11 mm) PAI region. We also limited the comparisons to ROIs within the same image, so as to mitigate the effects of inter-site and inter-patient variations [33]. Our sample pool did not include tissue with submucosal invasion. However, we consistently acquired PAI data beyond $2 \mathrm{~mm}$ from the tissue surface, which supports the imaging depth potential of PAI. Future studies will include samples with submucosal invasion. We note, however, that we have successfully imaged PAl-active nanoparticles lying below full-thickness ex vivo porcine intestine that was $2 \mathrm{~mm}$ thick (data not shown).

As regards the main clinical findings, the successful identification of BE relative to squamous mucosa indicated that the experimental protocol and image analysis used were not techni- 

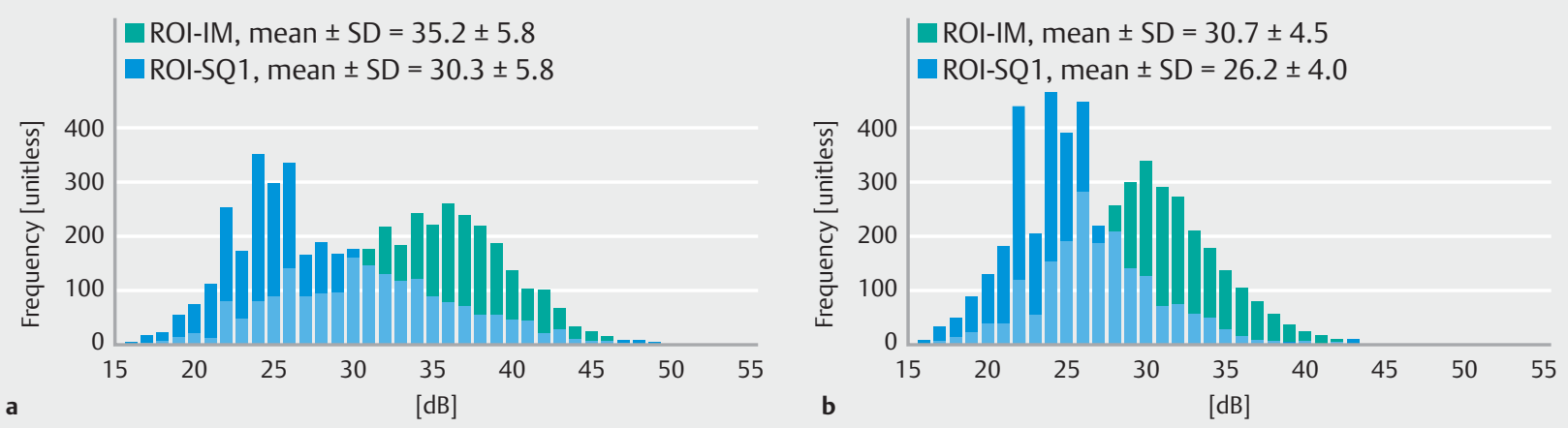

HbTot Distributions dB, Wil. Rank. Sum: $H=1, p=0$

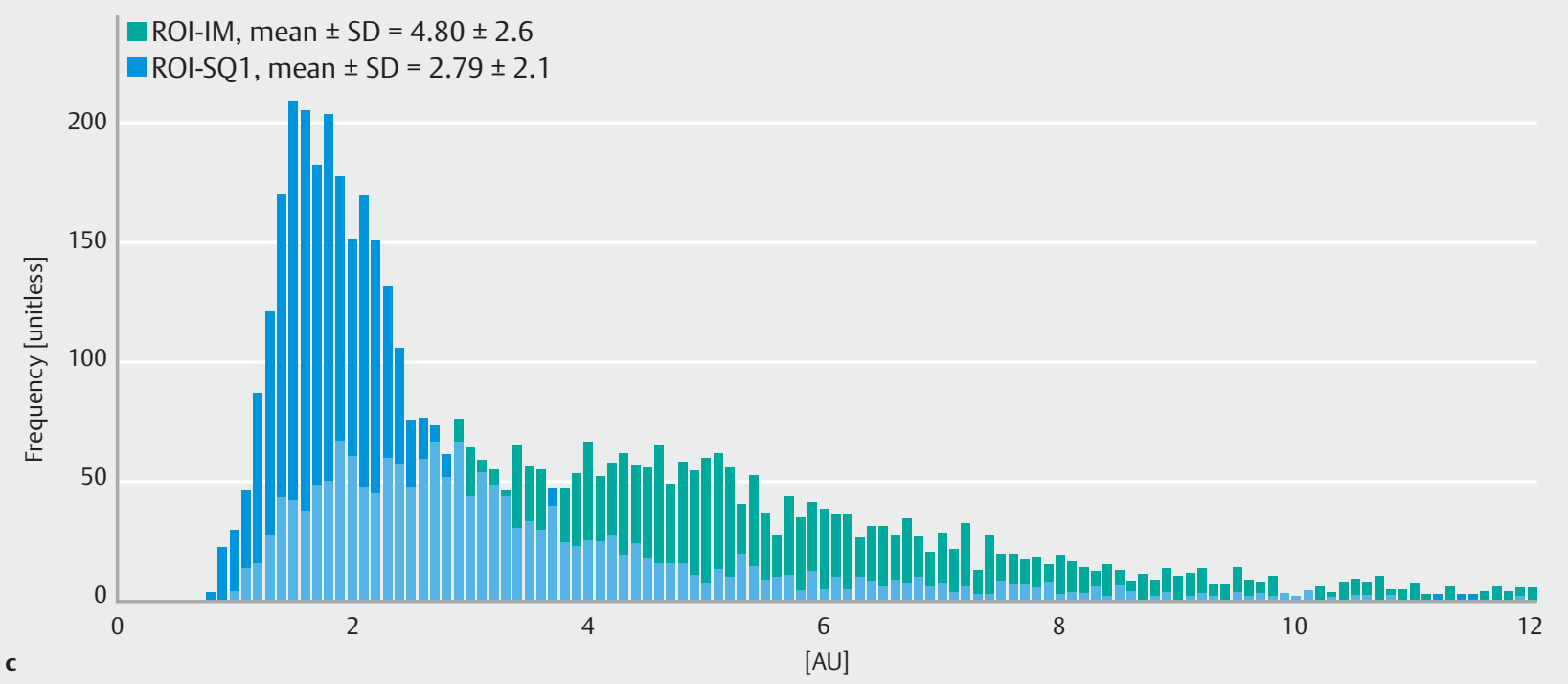

- Fig. 2 Example of frequency histograms of photoacoustic imaging intensity for two of the regions of interest (ROIs) shown in $>$ Fig. $1 \mathbf{b}$, corresponding to the middle green ROI (intestinal metaplasia [IM]) and left blue ROI (squamous mucosa [SQ1). a $750 \mathrm{~nm}$. b $850 \mathrm{~nm}$. c Relative total hemoglobin.

cally flawed. Furthermore, we repeated the comparison analysis using only the intrinsic PAI intensity (detailed in Supplementary Material S2) and this did not substantially change the results. The observations remained consistent at the ROI, slice, sample, and patient levels, indicating that the findings are not patient- or sample-biased (detailed in Supplementary Material S3). We realized our sample size was less than ideal, but it did serve the purposes of this first feasibility study (power and sample size analysis in Supplementary Material S4).

One might have expected the performance of PAI in identifying BE vs. squamous mucosa to be even better, given how distinct BE appears visually on endoscopy. However, there is very high heterogeneity in $\mathrm{BE}$, and not all regions are equally inflamed and hypervascularized. The more important point was the inability, at least in this ex vivo study, to reliably distinguish dysplasia from NDBE, which was hypothesized to be possible based on altered microvascularity. However, it should be noted that there is a relatively poor concordance between even expert
GI pathologists in identifying dysplasia, particularly low grade, in BE owing to considerable histological overlap between inflammatory/reactive changes and premalignant transformation [34].

A further limitation in interpreting the present results is that the ex vivo PAI data may not translate directly to the in vivo setting, primarily because of blood loss from the EMR tissue sample. Although most of the blood content is preserved by the ligation process, the blood does not necessarily fully fill the full vascular volume and there may be redistribution within the sample. The oxygen saturation $\left(\mathrm{SO}_{2}\right)$ value was omitted from the analysis to account for probable hypoxia post resection. Douplik et al. reported an algorithm using diffuse reflectance spectroscopy that involved a combination of the $\mathrm{Hb}_{\mathrm{T}}$ and $\mathrm{SO}_{2}$ values [10], so that accessing the $\mathrm{SO}_{2}$ information with in vivo $\mathrm{PAl}$ at multiple wavelengths may improve the diagnostic accuracy and, in the best-case scenario, result in clinically useful detection of dysplasia. To this end, we are developing an endo- 

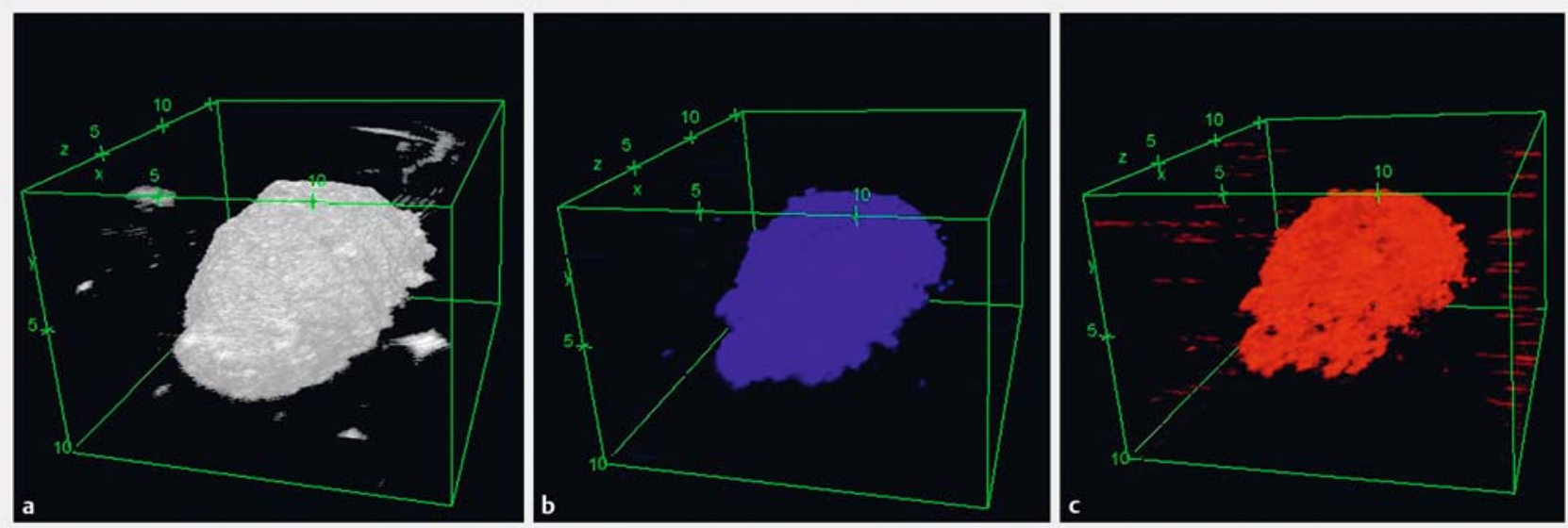

Fig. 3 3D reconstruction of the photoacoustic imaging (PAI) signal. a Ultrasound B-mode (grayscale). b PAl at $750 \mathrm{~nm}$ (blue), mainly dominated by hemoglobin absorption. c PAl at $850 \mathrm{~nm}$ (red), mainly dominated by oxy-hemoglobin absorption. Axis scale in mm.

- Table 1 Summary of region-of-interest comparisons of relative total hemoglobin between the subclasses of Barrett's esophagus.

\begin{tabular}{|c|c|c|c|c|c|c|}
\hline \multirow[t]{2}{*}{$H b_{T}$} & \multicolumn{2}{|c|}{ Dysplasia vs. NDBE } & \multirow{2}{*}{$\begin{array}{l}\text { NDBE } \\
\text { Inflammation* } \\
>\text { IM }\end{array}$} & \multicolumn{3}{|l|}{ BE vs. Squamous } \\
\hline & $\begin{array}{l}\text { Dysplasia } \\
>\text { Inflammation* }\end{array}$ & $\begin{array}{l}\text { Dysplasia } \\
>\mathrm{IM}\end{array}$ & & $\begin{array}{l}\text { Inflammation* } \\
\text { >Squamous }\end{array}$ & $\begin{array}{l}\text { Dysplasia } \\
\text { >Squamous }\end{array}$ & $\begin{array}{l}\text { IM } \\
\text { > Squamous }\end{array}$ \\
\hline Positive & 6 & 4 & 8 & 20 & 9 & 13 \\
\hline Negative & 12 & 8 & 19 & 4 & 1 & 3 \\
\hline Total & 18 & 12 & 27 & 24 & 10 & 16 \\
\hline
\end{tabular}

scopic PAI system and will use it to conduct an in vivo trial in the near future to confirm or alter the negative conclusion of the present study. A prototype endoscopic PAI instrument has been reported by Yang et al. [30], but to our knowledge the device has been used only in preclinical in vivo models in the esophagus. Even if intrinsic PAI is not adequate for detecting dysplasia, it should still have value in endoscopic staging of disease, because of its imaging depth capability.

Finally, if the intrinsic differences in microvascularity of dysplasia in BE does indeed prevent adequate endoscopic detectability using intrinsic PAI, then photoacoustic contrast agents may be a useful addition. Hence, in parallel, we are investigating the utility of PAI in combination with nontargeted and biomarker-targeted porphyrin-lipid nanoparticles (porphysomes) $[25,26]$, which may be administered systemically or by topical application to the tissue to minimize cost and potential toxicity.

\section{Acknowledgments}

This work was supported by an Emerging Frontiers grant (\#1022) from the Terry Fox Research Institute. The authors also thank Visualsonics Inc. for equipment support.
Competing interests

None

References

[1] Canada Cancer Society. Canadian Cancer Statistics 2014. Available from: http://www.cancer.ca/en/support-and-services/resources/ publications/ Accessed 24 Feb 2015

[2] American Cancer Society. Cancer Facts \& Figures 2015. Available from: https://www.cancer.org/research/cancer-facts-statistics/allcancer-facts-figures/ Accessed 24 Feb 2015

[3] Solaymani-Dodaran M, Logan RFA, West J et al. Risk of oesophageal cancer in Barrett's oesophagus and gastro-oesophageal reflux. Gut 2004; 53: $1070-1074$

[4] Shaheen NJ, Richter JE. Barrett's oesophagus. Lancet 2009; 373: 850 861

[5] Schnell TG, Sontag S], Chejfec G et al. Long-term nonsurgical management of Barrett's esophagus with high-grade dysplasia. Gastroenterology 2001; 120: $1607-1619$

[6] Reid BJ, Levine DS, Longton G et al. Predictors of progression to cancer in Barrett's esophagus: baseline histology and flow cytometry identify low- and high-risk patient subsets. Am J Gastroenterol 2000; 95: $1669-1676$ 
[7] Vennalaganti PR, Naag KanakadandiV, Gross SA et al. Inter-observer agreement among pathologists using wide-area transepithelial sampling with computer-assisted analysis in patients with Barrett's esophagus. Am J Gastroenterol 2015; 110: 1257-1260

[8] Haisley KR, Dolan JP, Olson SB et al. Sponge sampling with fluorescent in situ hybridization as a screening tool for the early detection of esophageal cancer. J Gastrointest Surg 2017; 21: 215-221

[9] Mannath J, Ragunath K. Role of endoscopy in early oesophageal cancer. Nat Publ Gr 2016; 13: $720-730$

[10] Douplik A, Zanati S, Saiko G et al. Diffuse reflectance spectroscopy in Barrett's Esophagus: developing a large field-of-view screening method discriminating dysplasia from metaplasia. J Biophotonics 2014; 7: 304-311

[11] Kara M, DaCosta RS, Wilson BC et al. Autofluorescence-based detection of early neoplasia in patients with Barrett's esophagus. Dig Dis 2004; 22 : $134-141$

[12] Messmann H, Endlicher E, Freunek $G$ et al. Fluorescence endoscopy for the detection of low and high grade dysplasia in ulcerative colitis using systemic or local 5-aminolaevulinic acid sensitisation. Gut 2003; 52: $1003-1007$

[13] Bergholt MS, Zheng W, Lin K et al. In vivo diagnosis of esophageal cancer using image-guided Raman endoscopy and biomolecular modeling. Technol Cancer Res Treat 2011; 10: 103-112

[14] Yang VXD, Tang SJ, Gordon ML et al. Endoscopic Doppler optical coherence tomography in the human GI tract: Initial experience. Gastrointest Endosc 2005; 61: 879-890

[15] Bouma B, Tearney G. High-resolution imaging of the human esophagus and stomach in vivo using optical coherence tomography. Gastrointest Endosc 2000; 51: $467-474$

[16] Singh R, Shahzad MA, Tam W et al. Preliminary feasibility study using a novel narrow-band imaging system with dual focus magnification capability in Barrett's esophagus: Is the time ripe to abandon random biopsies? Dig Endosc 2013; 25: 151 - 156

[17] Georgakoudi I, Jacobson BC, Van Dam J et al. Fluorescence, reflectance, and light-scattering spectroscopy for evaluating dysplasia in patients with Barrett's esophagus. Gastroenterology 2001; 120: $1620-1629$

[18] Raica M, Cimpean AM, Ribatti D. Angiogenesis in pre-malignant conditions. Eur J Cancer 2009; 45: 1924-1934

[19] Hanahan D, Folkman J. Patterns and emerging mechanisms of the angiogenic switch during tumorigenesis. Cell 1996; 86: 353-364

[20] Kim C, Erpelding TN, Jankovic L et al. Deeply penetrating in vivo photoacoustic imaging using a clinical ultrasound array system. Biomed Opt Express 2010; 1: 278-284
[21] Xu M, Wang LV. Photoacoustic imaging in biomedicine. Rev Sci Instrum 2006; 77: 41101

[22] Lim L, Streutker C], Marcon N et al. Clinical study of ex vivo photoacoustic imaging in endoscopic mucosal resection tissues. Proc SPIE 2015; 9323: 932307

[23] Needles A, Heinmiller A, Sun J et al. Development and initial application of a fully integrated photoacoustic micro-ultrasound system. IEEE Trans Ultrason Ferroelectr Freq Control 2013; 60: 888-897

[24] Conio M, Ponchon T, Blanchi S et al. Endoscopic mucosal resection. Am J Gastroenterol 2006; 101: 653-663

[25] Lovell JF, Jin CS, Huynh E et al. Porphysome nanovesicles generated by porphyrin bilayers for use as multimodal biophotonic contrast agents. Nat Mater 2011; 10: 324-332

[26] Ng KK, Shakiba M, Huynh E et al. Stimuli-responsive photoacoustic nanoswitch for in vivo sensing applications. ACS Nano 2014; 8: $8363-$ 8373

[27] Laser Institute of America. American national standard for safe use of lasers ANSI Z136.1-2014. Orlando: Laser Inst Am; 2014

[28] Laufer ], Elwell C, Delpy D et al. In vitro measurements of absolute blood oxygen saturation using pulsed near-infrared photoacoustic spectroscopy: accuracy and resolution. Phys Med Biol 2005; 50: $4409-4428$

[29] Wang X, Xie X, Ku G et al. Noninvasive imaging of hemoglobin concentration and oxygenation in the rat brain using high-resolution photoacoustic tomography. J Biomed Opt 2006; 11: 24015

[30] Maslov K, Sivaramakrishnan M, Zhang HF et al. Technical considerations in quantitative blood oxygenation measurement using photoacoustic microscopy in vivo. Proc SPIE, Photons Plus Ultrasound Imaging Sens 2006; 6086: 60860R1

[31] Cox BT, Arridge SR, Beard PC. Estimating chromophore distributions from multiwavelength photoacoustic images. J Opt Soc Am A Opt Image Sci Vis 2009; 26: $443-455$

[32] Laufer J, Delpy D, Elwell C et al. Quantitative spatially resolved measurement of tissue chromophore concentrations using photoacoustic spectroscopy: application to the measurement of blood oxygenation and haemoglobin concentration. Phys Med Biol 2007; 52: 141-168

[33] Lim L, Nichols B, Migden MR et al. Clinical study of noninvasive in vivo melanoma and nonmelanoma skin cancers using multimodal spectral diagnosis. J Biomed Opt 2014; 19: 117003

[34] Kerkhof M, Van Dekken H, Steyerberg EW et al. Grading of dysplasia in Barrett's oesophagus: substantial interobserver variation between general and gastrointestinal pathologists. Histopathology 2007; 50 : 920-927

[35] Yang J-M, Li C, Chen R et al. Catheter-based photoacoustic endoscope. J Biomed Opt 2014; 19: 66001 


\section{Supplementary Materials}

\section{S1. Patient List}

- TableS1 Detailed list of pre-EMR diagnosis, endoscopic descriptions, and resected specimens' diagnosis.

\begin{tabular}{|c|c|c|c|c|c|}
\hline $\begin{array}{l}\text { Patient } \\
\text { ID\# }\end{array}$ & Pre-EMR diagnosis & Endoscopic descriptions & $\begin{array}{l}\text { Paris Classi- } \\
\text { fication }\end{array}$ & $\begin{array}{l}\text { EMR } \\
\text { ID\# }\end{array}$ & $\begin{array}{l}\text { EMR diagnosis } \\
\text { (Staging-Stolte Classification) }\end{array}$ \\
\hline \multirow[t]{2}{*}{2} & \multirow[t]{2}{*}{$\begin{array}{l}\text { Suspicious for intramucosal } \\
\text { carcinoma }\end{array}$} & \multirow[t]{2}{*}{ Nodular lesion } & \multirow[t]{2}{*}{0 -Is } & 1 & $\begin{array}{l}\text { Adenocarcinoma } \\
\text { (T1M2) }\end{array}$ \\
\hline & & & & 2 & IM \\
\hline \multirow[t]{2}{*}{3} & \multirow{2}{*}{$\begin{array}{l}\text { Inflamed mucosa, no IM or } \\
\text { dysplasia identified }\end{array}$} & \multirow[t]{2}{*}{ Nodular GEJ } & \multirow[t]{2}{*}{$0-1 \mathrm{~s}$} & 6 & NDBE \\
\hline & & & & 12 & LGD \\
\hline 4 & HGD & Nodular BE & $0-1 \mathrm{~s}$ & 1 & HGD \\
\hline \multirow[t]{2}{*}{11} & \multirow[t]{2}{*}{ Intramucosal carcinoma } & \multirow[t]{2}{*}{ Irregular esophageal mucosa } & \multirow[t]{2}{*}{0 -lla } & 4 & LGD \\
\hline & & & & 5 & LGD \\
\hline 15 & LGD & $\begin{array}{l}\text { Post-ablation, area nonepithelia- } \\
\text { lized, slightly nodular }\end{array}$ & 0 -lla & 1 & NDBE \\
\hline \multirow[t]{2}{*}{16} & \multirow[t]{2}{*}{ Intramucosal carcinoma } & \multirow[t]{2}{*}{ Irregular slightly raised mucosa } & \multirow[t]{2}{*}{0 -Ila } & 1 & Adenocarcinoma (T1M2)+HGD \\
\hline & & & & 3 & HGD \\
\hline \multirow[t]{2}{*}{17} & \multirow[t]{2}{*}{ HGD } & \multirow{2}{*}{$\begin{array}{l}\text { Slight nodularity and erosion at } \\
\text { GEJ }\end{array}$} & \multirow[t]{2}{*}{0 -Ila; 0 -Ilc } & 1 & NDBE \\
\hline & & & & 2 & NDBE \\
\hline 18 & HGD & Slightly raised mucosa & $0-I l a$ & 1 & $\begin{array}{l}\text { Adenocarcinoma } \\
(\mathrm{T} 1 \mathrm{M} 4)+\mathrm{HGD}\end{array}$ \\
\hline
\end{tabular}

\section{S2. PAI Comparison Analysis using PAI intensity}

We repeated the region-of-interest (ROI) analysis using the PAI intensity at $680 \mathrm{~nm}$ (maximum laser output) as well as at 750 and $850 \mathrm{~nm}$. In the case shown in > Fig. 1a,b and > Fig. 2, we considered the photoacoustic intensity of ROI-1 to be higher than that of ROI-2 (i.e. to have a "positive" score) when 2 or 3 variables have higher mean value and the difference in their frequency distributions is statistically significant $(P<0.05)$. $>$ Table $\mathbf{2}$ summarizes the corresponding comparisons using all 3 wavelengths. Overall, the findings results did not change significantly from the use of the relative $\mathrm{Hb}_{\mathrm{T}}$ values, so that $\mathrm{Hb}_{\mathrm{T}}$ adequately represents the collected data.

- Table S2 Summary of ROI comparison analysis using majority poll of PA intensity.

\begin{tabular}{|c|c|c|c|c|c|c|}
\hline \multirow[t]{2}{*}{ Score } & \multicolumn{2}{|c|}{ Dys vs. NDBE } & \multirow{2}{*}{$\begin{array}{l}\text { NDBE } \\
\text { InfI > IM }\end{array}$} & \multicolumn{3}{|l|}{ BE vs. Squ } \\
\hline & Dys $>$ Infl & Dys $>I M$ & & Infl $>$ Squ & Dys $>$ Squ & $\mathrm{IM}>\mathrm{Squ}$ \\
\hline Positive & 6 & 4 & 13 & 20 & 9 & 14 \\
\hline Negative & 12 & 8 & 14 & 4 & 1 & 2 \\
\hline Total & 18 & 12 & 27 & 24 & 10 & 16 \\
\hline
\end{tabular}




\section{S3. Comparison Level: ROI vs. Slice vs. Sample vs. Patient}

We acquired data from at least one tissue sample from each patient. Each sample can have multiple slices, depending on the size. Within each slice, multiple ROI comparisons are possible, depending on the number of permutations between ROls. One potential problem is then that the results could be biased by a subgroup of tissue samples or patients if these contributed a major fraction of the data. To check this, we carried out the comparison analysis at each of the ROI, tissue slice, tissue sample, and patient levels. At the ROI level, each comparison was counted as an observation. At the tissue slice level, ROI comparisons from the same slice were combined, with the majority of the observations determining the outcome for that slice. At the tissue sample level, observations from all slices from the sample were combined, and at the patient level observations from all the tissue sample(s) were combined. The most important comparisons are summarized in $>$ Table $\mathbf{S 3}$.

Again, the relative $\mathrm{Hb}_{\mathrm{T}}$ in $\mathrm{BE}$ was higher than in squamous esophagus at least $75 \%$ of the time at every level, whereas it was not consistent at any level between dysplasia and NDBE.

\section{S4. Power and Sample Size Analysis}

Notwithstanding the small number of patients in this feasibility study, we conducted a power analysis to compare the mean difference between ROIs. With expected 50 observations (at least 50 data points/pixels for each ROI) in each ROI, we have at least $90 \%$ power to detect significant difference between the two ROIs. The calculation is based on the assumed effect size (mean difference) at 1 SD. In order to adjust for multiple comparisons, the significance level is set at $0.0005(0.05 / 100$, as we conducted 107 tests in total).

We also conducted sample size calculation for a future pilot study. If the power for a single sample test is 0.90 as we conducted above, a total sample size of 35 EMR samples can produce a two-sided $95 \%$ confidence interval with a width equal to $0.2(0.80-1.00)$.

- TableS3 Comparison analysis at different levels.

\begin{tabular}{|c|c|c|c|c|c|}
\hline & & ROI & Slice & Sample & Patient \\
\hline \multirow[t]{4}{*}{ Dys vs. NDBE } & Positive & 10 & 3 & 1 & 0 \\
\hline & Negative & 20 & 5 & 4 & 3 \\
\hline & Same & 0 & 3 & 0 & 1 \\
\hline & Total & 30 & 11 & 5 & 4 \\
\hline \multirow[t]{4}{*}{ BE vs. Squ } & Positive & 42 & 12 & 6 & 5 \\
\hline & Negative & 8 & 3 & 2 & 1 \\
\hline & Same & 0 & 1 & 0 & 0 \\
\hline & Total & 50 & 16 & 8 & 6 \\
\hline
\end{tabular}

Dys, dysplasia; NDBE, nondysplastic Barrett's esophagus; BE, Barrett's esophagus; Squ, squamous; ROI, region of interest. 